\section{A combination of tests done in general practice could assess the risk for left ventricular systolic dysfunction}

Nielsen OW,Hansen JF, Hilden J, et al. Risk assessment of left ventricular systolic dysfunction in primary care: cross

sectional study evaluating a range of diagnostic tests. BMJ 2000 Jan 22;320:220-4.

QUESTION: In patients at increased risk for left ventricular systolic dysfunction (LVSD), are there clinical methods that general practitioners can use to determine which patients to refer for echocardiography?

\section{Design}

Blinded comparison of clinical data with echocardiographic results.

\section{Setting}

3 general practices in Copenhagen, Denmark.

\section{Patients}

126 patients who were 49 to 93 years of age (median age $71 \mathrm{y}, 56 \%$ women) and had past or present signs or symptoms of heart disease, treatment for heart disease, referral to a coronary care unit, or previous cardiac abnormalities. Nursing home patients and all patients actively treated for advanced heart failure were excluded.

\section{Description of tests and diagnostic standard}

All patients received a physical examination, chest radiography, echocardiography, supine 12 lead electrocardiography, blood pressure measurements, and blood tests. LVSD was defined as an ejection fraction of $<0.45$ and was determined from videotapes and photoechocardiograms by an investigator blinded to the other clinical data.

\section{Main outcome measures}

Sensitivity, specificity, and likelihood ratios of clinical data for detecting LVSD.

\section{Main results}

Resting supine heart rate > supine diastolic blood pressure, $N$-terminal atrial natriuretic peptide $(N$-ANP) level $>0.8 \mathrm{nmol} / \mathrm{L}$, and abnormal electrocardiographic (ECG) results were the signs and symptoms significantly results were the most useful for ruling out systolic dysfunction.

\section{Conclusions}

A combination of heart rate $>$ diastolic pressure, $N$-terminal atrial natriuretic peptide level $>0.8 \mathrm{nmol} / \mathrm{l}$, and abnormal electrocardiographic test results were accurate in determining who to refer for echocardiography to identify systolic dysfunction. No single test could diagnose left ventricular systolic dysfunction, but normal electrocardiographic test results could help to rule it out. associated with LVSD ( $\mathrm{s} \leqslant 0.006)$ (table). Normal ECG

\section{COMMENTARY}

It is important to identify patients with symptoms of congestive heart failure who have LVSD because their survival can be lengthened and symptoms alleviated by the medical armamentarium. It is equally important to rule out the diagnosis of LVSD to avoid treatment with medications that are potentially harmful in other conditions (eg, digoxin in the case of diastolic dysfunction or $\beta$-adrenergic blocking agents for pulmonary disease).

2 possible approaches exist for identifying symptomatic patients with LVSD: echocardiography in all presenting patients and echocardiography in a high risk subset of patients. The former is common practice because of the implications of missing the diagnosis. Nielsen et al studied 3 indices that might identify the subset of patients who have signs or symptoms of heart disease and who are most likely to have LVSD: electrocardiography, which is sensitive but not specific for $\mathrm{LVSD}^{1} ; \mathrm{N}$-ANP, which reflects increased atrial pressure and distention and is part of the neurohumoral compensation to systolic dysfunction; and heart rate $>$ diastolic blood pressure, which is a new, arbitrary index that presumably reflects the sympathetic nervous system's compensation for systolic dysfunction. They found that if abnormal ECG results alone are used for risk stratification, 60 of 120 echocardiograms would be avoided but at the cost of missing the diagnosis of LVSD in 1 patient. When echocardiography is done only in patients with abnormal ECG results and either an increased $N$-ANP or heart rate $>$ diastolic pressure, 100 of 120 tests would be avoided but at the cost of missing the diagnosis in 4 patients. The study does not mention the clinical utility of echocardiography for determining the cause of LVSD or for assessing such concomitant disorders as valvular disease. Thus, withholding echocardiography in patients at lower risk for LVSD might avoid performance of a large number of tests giving negative results but would fail to reach the correct diagnosis in a substantial number of patients with LVSD. The next step in deciding on the optimal strategy is to do a controlled study of a larger number of patients and a cost benefit analysis.

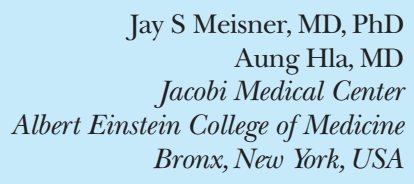

1 Davie AP, Francis CM, Love MP, et al. Value of the electrocardiogram in identifying heart failure due to left ventricular systolic dysfunction. BMJ 1996;312:222.

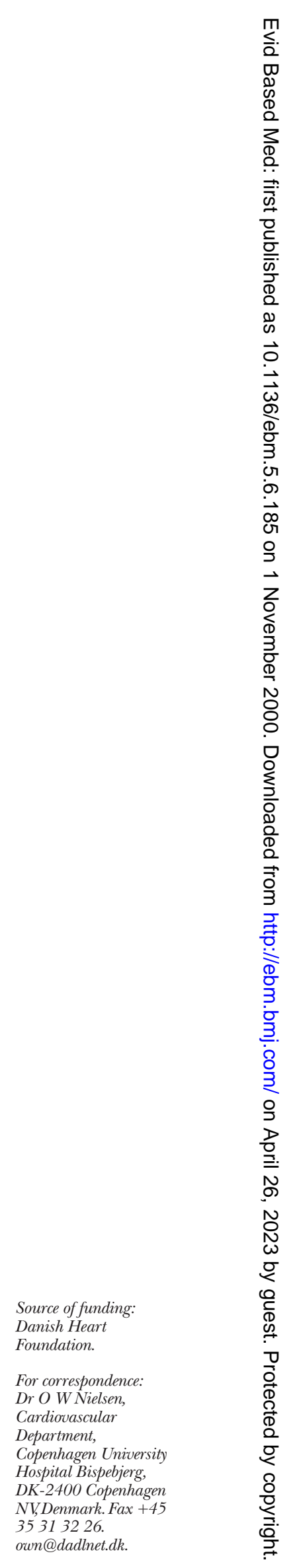

Characteristics of tests to detect left ventricular systolic dysfunction*

\begin{tabular}{lllll} 
Test characteristics & Sensitivity (95\% Cl) & Specificity (CI) & +LR & -LR \\
Abnormal electrocardiographic results & $87 \%(60$ to 98$)$ & $56 \%(46$ to 65$)$ & 1.96 & 0.24 \\
\hline$N$-terminal atrial natriuretic peptide level $>0.8 \mathrm{nmol} / \mathrm{l}$ & $43 \%(18$ to 71$)$ & $89 \%(81$ to 94$)$ & 3.82 & 0.62 \\
\hline Heart rate $>$ diastolic pressure & $53 \%(27$ to 79$)$ & $86 \%(79$ to 92$)$ & 3.91 & 0.55 \\
\hline
\end{tabular}

*Abbreviations defined in glossary; +LR and Cls calculated from data in article. 